\title{
Group B Streptococci Investigation in Pre-term Labors
}

\author{
Elahe Zarean Seyyed, Ehsan Toossi, Akram Jalalvand, Mehdi Sajadi \\ Isfahan University of Medical Sciences, Isfahan, Iran
}

ackground: Pre-term delivery is still a main cause of perinatal mortality and morbidities. This study D was designed to find any association between maternal Group B Streptococcus (GBS) colonization and pre-term delivery. Method: This case-control study was conducted from March 2010 to May 2011 at two referral university hospitals (Al-Zahra and Shaheed Beheshty), Isfahan University of Medical Sciences, Isfahan, Iran. The study sample of pregnant women was composed of a control group of 200 term pregnancy with at least 37 complete weeks, and a case group of another 200 women between 20 to 37 weeks of pregnancy with pre-term labor. Maternal GBS colonization was compared between two groups. Results: Maternal age ( $26.03 \pm 4.81$ vs. $27.19 \pm 5.60, \mathrm{P}=0.07)$ and gravid $(1.75 \pm 0.88$ vs. $1.83 \pm 1.08, \mathrm{P}=0.52)$ were similar in case and control groups. Level of education and gestational age were significantly higher in control versus case group $(\mathrm{P}=0.01, \mathrm{P}=0.00)$. History of antibiotic use during pregnancy was more frequent in case group compared with control group $(\mathrm{P}=0.00)$. There were $59 / 151$ (39.1\%) positive vaginal samples in case group compared with $36 / 178(20.2 \%)$ in control group $(\mathrm{P}=0.000)$. There were $55 / 151$ (36.4\%) positive rectal samples in case group versus 30/178 (16.9\%) in control group ( $\mathrm{P}=0.000)$. Conclusion: This study showed that GBS colonization was statistically higher in pre-term labors compared with term deliveries. To understand the mechanism of GBS effect on pregnancy, large observational studies are needed, with clearly defined outcomes, and with prognostic risk factors for pre-term delivery taken into account. Key words: Pre-term Labor; Group B Streptococcus; Recto vaginal.

Corresponding author: Akram Jalalvand, MD, School of Medicine, Isfahan University of Medical Sciences, Isfahan, Iran E-mail: akramjalalvand@gmail.com

\section{INTRODUCTION}

Pre-term delivery is still a main cause of perinatal mortality and morbidities. The evidences showed the rates of pre-term labor increased from $9.7 \%$ in 1990 to more than $10.8 \%$ in 2004 in the developed countries (1, 2). In Iran, the rate of pre-term labors was estimated about $13.9 \%$ (3). These data highlight the importance of identifying pregnant women at a risk of pre-term labor and the need to develop prevention strategies.

Pre-term labor has already been shown to be associated with some sociodemographic and pathological factors, such as low socioeconomic level, genitourinary tract infection $(4,5)$. However, the mechanisms of pre-term labor have been controversial yet. Maternal genital tract coloni- zation with specific organisms can play a role in pre-term delivery. Bacterial products such as phospholipases $A_{2}$ and $C$, endotoxin, and induction of the cytokine cascade can stimulate the prostaglandin pathway and initiate labor $(1,2)$. Even in nonpregnant women, the presence of pathogens in the genitourinary tract has been shown to induce cytokine production (6, 7). Therefore, the mechanism by which the presence of genitourinary infections may lead to pre-term labor seems to be related to the immune system. Up to $36 \%$ of pregnant women are colonized with Group B Streptococcus (GBS), most often without having symptoms $(6,8)$. In Iran, the rate of GBS colonized mother was estimated about $15-18 \%(9,10)$. Pre-term delivery in GBS col- onized mothers is a recognized risk factor for early onset neonatal GBS disease (GBSEOD) (11), but whether maternal GBS genital colonization is related to pre-term delivery is unclear. This study was designed to find any association between maternal GBS colonization and pre-term delivery.

\section{METHODS AND MATERIALS}

This case-control study was conducted from March 2010 to May 2011 at two referral university hospitals (Al-Zahra and Shaheed Beheshty), Isfahan University of Medical Sciences, Isfahan, Iran. The study sample of pregnant women was composed of a control group of 200 term pregnancy with at least 37 complete weeks, and a case group of another 200 women between 20 to 37 weeks of pregnancy with pre-term labor. Exclusion criteria were hypertension, cardiovascular diseases, placenta abruption, Pre-term Premature Rupture of Membranes (PPROM), multi fetal pregnancy and the use of antibiotics during the two preceding weeks. The diagnosis of pre-term labor was documented according to contractions of four in 20 minutes or eight in 60 minutes plus progressive change in the cervix, or cervical dilatation greater than $1 \mathrm{~cm}$ or cervical effacement of 80 percent or greater (12). The Ethics Committee of the Isfahan University of Medical Sciences approved the study protocol and informed consent was obtained from all women after full explanation of the study aims and protocol. After registering demographic data such as maternal and gestational age, gravid, level of education and history of antibiotic use during pregnancy, women underwent a speculum 
examination at which two vaginal and rectal swabs were collected for investigation of GBS.

Samples were collected from the proximal third of the vaginal introitus and from inside the anus through the anal sphincter. Two swabs (one vaginal and one anorectal) were separately inoculated into two tubes containing Todd-Hewitt enrolled in the study broth (manufactured by Alpha Biosciences, Inc. Maryland, USA), supplemented with gentamicin and nalidixic acid immediately and incubated for 24 hours, and then subcultured for 24 hours on non-selective $5 \%$ blood agar plates. The final identification of GBS in all samples was made by the CAMP test, which is based on the production of an arrow-shaped hemolysis zone in conjunction with a Staphylococcus aureus colony in a blood-agar plate. Detection rates and each sampling site were calculated. Chi-square and independent tests were used to detect significant differences, and a P value $<0.05$ was considered statistically significant.

\section{RESULTS}

Of 200 women in case group, 49 women were excluded in order to PPROM. Of 200 women in control group, 22 women were excluded in order to PROM. One hundred and fifty one women in case group and 178 women in control group were enrolled in the study. The mean of maternal age was $26.03 \pm 4.81$ years (range: $17-36$ years) in case group and $27.19 \pm 5.60$ years (range: 17-42) in control group. Demographic and obstetric characteristics of the women enrolled in the study were shown in Table 1. There were 8 (5.3\%) women in case group and $2(1.1 \%)$ women in control group who had not answered to level of education. Twenty four (15.9\%) women in case group and 6 (3.4\%) in control group were not assured about the antibiotic using during pregnancy. Therefore, they were excluded from final analysis of these factors. There were 59/151 (39.1\%) positive vaginal samples in case group compared with $36 / 178$ (20.2\%) in control group. According to Chisquare test there was statistical difference between two groups $(\mathrm{P}=0.000)$. There were $55 / 151$ (36.4\%) positive rectal samples in case group versus $30 / 178$ (16.9\%) in con- trol group. According to Chi-square test there was statistical difference between two groups $(\mathrm{P}=0.000)$. All women with positive rectal samples had positive vaginal results in investigation of GBS.

\section{DISCUSSION}

This study showed that GBS colonization was statistically higher in pre-term labors compared with term deliveries. Lower level of education and positive history of antibiotic use during pregnancy were related to higher prevalence of pre-term labor. Investigation of GBS colonization for all pregnant women between 35 and 37 weeks is recommended, and in situations of risk of pre-term delivery, which are pre-term labor and pre-term premature rupture of membranes (13). Evidences suggested antibiotic prophylaxis of colonized women, during labor, greatly reduces the risk of neonatal disease (14). Previous studies showed GBS isolation is $20 \%$ to $40 \%$ greater when combined vaginal and anorectal cultures are collected (15-16). A significant proportion of women were found to have only one of these sites colonized; this proportion was $18 \%$ to $24 \%$ higher in anorectal compared to vaginal samples (16). Our results were not agreed with them. We found all women with positive rectal samples had positive vaginal results in investigation of GBS. This result may indicate that anorectal sampling is not necessary for screening of GBS. We used Todd-Hewitt enriched medium with gentamicin and nalidixic acid for investigating GBS to inhibit growth of Gram-negative bacteria, and it has greater sensitivity when compared to other nonselective media, such as blood or Granada agar (17). Our study showed positive GBS cultures more frequently in patients with pre-term delivery. Also we found that positive history of antibiotic use during pregnancy is related to higher frequencies of pre-term labor. It may be because of the change of vaginal microbial ecosystem due to antibiotic use. Our results indicated that level of education had a reverse relation with frequency of pre-term labors. This result is agreed with Nomura et al. (18) study which GBS colonization are more frequent than women who had lower level of education. The high sample size and being multicentric are strengths of our study. Not determining the another risk factors of pre-term labor such as cigarette smoking, use of cocaine, uterine malformation, and multi fetal gestation was limitation of our study.

\section{CONCLUSION}

This study showed that GBS colonization was statistically higher in pre-term labors compared with term deliveries. To understand the mechanism of GBS effect on pregnancy, large observational studies are needed, with clearly defined outcomes, and with prognostic risk factors for pre-term delivery taken into account.

\section{Acknowledgment}

This study was sponsored by Isfahan University of Medical Sciences with research project number 188053. We are thankful from Dr Hamidreza Shemshaki (Isfahan University of Medical Sciences) for editing our final draft.

\section{REFERENCES}

Nordenvall M, Sandstedt B. Chorioamnionitis in relation to gestational outcome in a Swedish population. Eur J Obstet Gynecol Reprod Biol. 1990; 36: 59-67.

Tucker J, McGuire W. Epidemiology of pre-term birth. BMJ. 200 18; 329: 675-678.

Ganji T, Einipour Z, Hosseini F. The Relationaship of Pre-Term Delivery and Stressful Life Events during Pregnancy. Iran I Nursing. 2009:21: 77-86.

Culhane JF, Iams JD, Romero R. Epidemiology and e-term birth. Lancet. 2008; 371: 75-84. bor and birth in the United States. J Midwifery Womens Health. 2007; 52: 281-290.

Basso B, Giménez F, López C. IL-13, IL-6 and IL-8 levels in gynecoobstetric infections. Infect Dis Obstet Gynecol. 2005; 13: 207-211 Jun JK, Yoon BH, Romero R, Kim M, Moon JB, Ki SH et al. Interleukin 6 determinations in cervical Xuid have diagnostic and prognosGynecol. 2000; 183: 868-873. Doh K, Barton PT, Korneeva I, Perni SC, Bongiovanni AM, Tut tle SL. et al. DiVerential vaginal expression of interleukin 1 sysplasma urealyticum in pregnant women. Infect Dis Obstet Gynecol. 2004; 12: 79-85.

9. Khataie GH, Shahrokhi N. Bacteriologic and serologic diagnosis of group B streptococci in pregnant women, neonates and infants. Teh-

ran Univ Med J. 1998: 56: 54-60.
10. Amirmozafari N, Mansour Ghanaei M, Sadr Nouri B, Farhadi Tooli L. Survey revalence of Group B Streptococci in genital tract women in 28-37 weeks pregnancy. crobial prophylaxis for prevention of group-B-streptococcal disease on the incidence and ecology of early-onset neonatal sepsis. Lancet Infect Dis. 2003; 3: 201-213. Cunningham F, Leveno K, Bloom S, Hauth J, Rouse D, Spong C. Williams Obstetrics. 23rd ed. New York: McGraw-Hill, 2010. Schrag S, Gorwitz R, Fultz-Buts K, Schuchat A. Prevention of pernatal group B streptococcal disease. Revised guidelines from $C D C$

MMWR 2002;51(RR-11): 1-22.
Schuchat A. Group B streptococcal disease: from trials and tribulations to triumph and trepidation. Clin Infect Dis. 2001; 33(6)

5. Jaureguy F, Carton M, Teboul J. et al. Risk factors and screenroup B streptococcal colonization in pregnant women: results of a prospective study. J Gynecol Obstet Biol Reprod. 2003; 32(2): 132-138.

16. Quinlan J.D, Hill DA, Maxwell BD. et al. The necessity of both anorectal and vaginal cultures for group B streptococcus screening during pregnancy. J Fam Pract. 2000; 49(5): 447-448. Gupta C, Briski LE. Comparison of two culture media and three sampling techniques for sensitive and rapid screening of vaginal colonization by group B streptococcus in pregnant women. J Clin M

8. Nomura ML, Passini Junior R, Oliviera UM. Selective versus NonPregnancies Complicated by Pre-term Labor or Preterm-Prematur Rupture of Membranes. BIID. 2006; 10: 247-250. 